\title{
L-DOPA cyclohexyl ester, an L-DOPA antagonist, protects neurostimmulated contractile response in mice heart
}

\author{
Tatsuo Hashimoto ${ }^{1}$, Shogo Hamaguchi ${ }^{2}$, Daiki Masukawa ${ }^{1}$, Motokazu Koga ${ }^{1}$, Masayuki Nakano ${ }^{1}$, \\ Hikaru Tanaka ${ }^{2}$, Kouichi Tamura ${ }^{3}$, Yoshio Goshima ${ }^{1}$ \\ ${ }^{I}$ Pharmacology, Yokohama City University School of Medicine, Japan, ${ }^{2}$ Pharmacology, Toho University Faculty of \\ Phamaceutical sciences, Japan, ${ }^{3}$ Cardiorenal Medicine, Yokohama City University School of Medicine, Japan
}

We previously showed that L-3,4-dihydroxyphenylalanine (L-DOPA), a precursor of catecholamines, sensitize the vascular adrenergic receptor, alpha 1 through activation of L-DOPA receptor GPR143 (JCI Insight, Masukawa et al., 2017). In wild-type mice, intravenous infusion of phenylephrine induced a transient elevation of blood pressure. This response was attenuated in Gpr143 gene-deficient (GPR143-KO) mice. In conditional knockout mice deficient in GPR143 in vascular smooth muscle cells (VSMCs), (sm22-cre;Gpr143fl/y), the pressor response to phenylephrine was attenuated, indicating that L-DOPA directly modulates alpha1-adrenergic receptor signaling in the VSMCs. Intravenous infusion of L-DOPA cyclohexyl ester (L-DOPA CHE), an L-DOPA antagonist, alone decreased the blood pressure in wild-type mice but not in whole-body Gpr143-KO mice. Unexpectedly, however, L-DOPA CHE-induced decrease in blood pressure was similarly observed in sm22-cre;Gpr143fl/y mice. This suggests that the target GPR143 responsible for the depressor action of L-DOPA CHE may reside in tissues other than VSMCs. In the present study, we investigated the effect of L-DOPA CHE on the functions of isolated right ventricle of mice. L-DOPA CHE at 30-100 uM concentrationdependently suppressed contractile response to sympathetic nerve stimulation. Our findings suggest that GPR143 may also regulate the responsiveness of the heart to sympathetic nerve stimulation. 